\title{
Recent results from the MAJORANA DEMONSTRATOR
}

\section{J. Myslik*, ${ }^{a}$ S.I. Alvis, ${ }^{b}$ I.J. Arnquist ${ }^{c}$ F.T. Avignone III, ${ }^{d e}$ A.S. Barabash, ${ }^{f}$}

C.J. Barton, ${ }^{g}$ F.E. Bertrand, ${ }^{e}$ T. Bode, ${ }^{h}$ B. Bos,${ }^{i}$ V. Brudanin,,${ }^{j}$ M. Busch, ${ }^{k l}$ M. Buuck, ${ }^{b}$ T.S. Caldwell, ${ }^{m l}$ Y-D. Chan, ${ }^{a}$ C.D. Christofferson, ${ }^{i}$ P.-H. Chu, ${ }^{n}$ C. Cuesta,${ }^{b \dagger}$ J.A. Detwiler, ${ }^{b}$ C. Dunagan, ${ }^{i}$ Yu. Efremenko, ${ }^{o e}$ H. Ejiri, ${ }^{p}$ S.R. Elliott, ${ }^{n}$ T. Gilliss, ${ }^{m l}$ G.K. Giovanetti, ${ }^{q}$ M.P. Green, ${ }^{r l e}$ J. Gruszko, ${ }^{s}$ I.S. Guinn, ${ }^{b}$ V.E. Guiseppe, ${ }^{d}$ C.R. Haufe, ${ }^{m l}$ R.J. Hegedus, ${ }^{m l}$ L. Hehn, ${ }^{a}$ R. Henning, ${ }^{m l}$ D. Hervas Aguilar, ${ }^{m l}$ E.W. Hoppe, ${ }^{c}$ M.A. Howe, ${ }^{m l}$ K.J. Keeter, ${ }^{t}$ M.F. Kidd, ${ }^{\prime}$ S.I. Konovalov, ${ }^{f}$ R.T. Kouzes, ${ }^{c}$ A.M. Lopez, ${ }^{o}$ R.D. Martin, ${ }^{v}$ R. Massarczyk, ${ }^{n}$ S.J. Meijer, ${ }^{m l}$ S. Mertens, ${ }^{h w}$ G. Othman, ${ }^{m l}$ W. Pettus, ${ }^{b}$ A. Piliounis, ${ }^{v}$ A.W.P. Poon, ${ }^{a}$ D.C. Radford, ${ }^{e}$ J. Rager, ${ }^{m l}$ A.L. Reine, ${ }^{m l}$ K. Rielage,${ }^{n}$ N.W. Ruof ${ }^{b}{ }^{b}$ B. Shanks,${ }^{e}$ M. Shirchenko, ${ }^{j}$ D. Tedeschi, ${ }^{d}$ R.L. Varner, ${ }^{e}$ S. Vasilyev, ${ }^{j}$ Vasundhara, ${ }^{v}$ B.R. White, ${ }^{n}$ J.F. Wilkerson, ${ }^{m l e}$ C. Wiseman, ${ }^{b}$ W. Xu, ${ }^{g}$ E. Yakushev, ${ }^{j}$ C.-H. Yu, ${ }^{e}$ V. Yumatov, ${ }^{f}{ }^{\prime}$. Zhitnikov, ${ }^{j}$ and B.X. Zhu ${ }^{n}$

${ }^{a}$ Nuclear Science Division, Lawrence Berkeley National Laboratory, Berkeley, CA, USA

${ }^{b}$ Center for Experimental Nuclear Physics and Astrophysics, and Department of Physics, University of Washington, Seattle, WA, USA

${ }^{c}$ Pacific Northwest National Laboratory, Richland, WA, USA

${ }^{d}$ Department of Physics and Astronomy, University of South Carolina, Columbia, SC, USA

e Oak Ridge National Laboratory, Oak Ridge, TN, USA

${ }^{f}$ National Research Center "Kurchatov Institute" Institute for Theoretical and Experimental Physics, Moscow, Russia

${ }^{g}$ Department of Physics, University of South Dakota, Vermillion, SD, USA

${ }^{h}$ Max-Planck-Institut für Physik, München, Germany

${ }^{i}$ South Dakota School of Mines and Technology, Rapid City, SD, USA

${ }^{j}$ Joint Institute for Nuclear Research, Dubna, Russia

${ }^{k}$ Department of Physics, Duke University, Durham, NC, USA

${ }^{l}$ Triangle Universities Nuclear Laboratory, Durham, NC, USA

${ }^{m}$ Department of Physics and Astronomy, University of North Carolina, Chapel Hill, NC, USA

${ }^{n}$ Los Alamos National Laboratory, Los Alamos, NM, USA

${ }^{\circ}$ Department of Physics and Astronomy, University of Tennessee, Knoxville, TN, USA

${ }^{p}$ Research Center for Nuclear Physics, Osaka University, Ibaraki, Osaka, Japan

${ }^{q}$ Department of Physics, Princeton University, Princeton, NJ, USA

${ }^{r}$ Department of Physics, North Carolina State University, Raleigh, NC, USA

${ }^{s}$ Department of Physics, Massachusetts Institute of Technology, Cambridge, MA, USA

${ }^{t}$ Department of Physics, Black Hills State University, Spearfish, SD, USA

"Tennessee Tech University, Cookeville, TN, USA

${ }^{v}$ Department of Physics, Engineering Physics and Astronomy, Queen's University, Kingston, ON, Canada

${ }^{w}$ Physik Department, Technische Universität, München, Germany

E-mail: jwmyslikelbl.gov 
The Majorana Demonstrator is an experiment constructed to search for neutrinoless double-beta decay in ${ }^{76} \mathrm{Ge}$ and to demonstrate the feasibility to deploy a large-scale experiment in a phased and modular fashion. It consists of two modules of natural and ${ }^{76} \mathrm{Ge}$-enriched germanium detectors totalling $44.1 \mathrm{~kg}$, operating at the 4850' level of the Sanford Underground Research Facility in Lead, South Dakota, USA. Commissioning of the experiment began in June 2015, followed by data production with the full detector array in August 2016. The ultra-low background and record energy resolution achieved by the MAJORANA DEMONSTRATOR enable a sensitive neutrinoless double-beta decay search, as well as additional searches for physics beyond the Standard Model. I will discuss the design elements that enable these searches, along with the latest results, focusing on the neutrinoless double-beta decay search. I will also discuss the current status and the future plans of the MAJORANA DEMONSTRATOR, as well as the plans for a future tonne-scale ${ }^{76} \mathrm{Ge}$ experiment.

This material is based upon work supported by the U.S. Department of Energy, Office of Science, Office of Nuclear Physics, the Particle Astrophysics and Nuclear Physics Programs of the National Science Foundation, the Russian Foundation for Basic Research, the Natural Sciences and Engineering Research Council of Canada, the Canada Foundation for Innovation John R. Evans Leaders Fund, the National Energy Research Scientific Computing Center, and the Oak Ridge Leadership Computing Facility, and the Sanford Underground Research Facility.

The 39th International Conference on High Energy Physics (ICHEP2018)

4-11 July, 2018

Seoul, Korea

\footnotetext{
* Speaker.

${ }^{\dagger}$ Present address: Centro de Investigaciones Energéticas, Medioambientales y Tecnológicas, CIEMAT 28040, Madrid, Spain
} 


\section{Introduction}

The Majorana Demonstrator [1] is a ${ }^{76} \mathrm{Ge}$ neutrinoless double-beta decay ( $0 v \beta \beta$-decay) experiment, currently operating at the 4850' level of the Sanford Underground Research Facility (SURF). It consists of $44.1 \mathrm{~kg}$ of p-type point contact germanium crystal detectors, $29.7 \mathrm{~kg}$ enriched to $88.1 \% \pm 0.7 \%{ }^{76} \mathrm{Ge}$. They are installed in two vacuum cryostats, shielded with copper, lead, and polyethylene, with an active muon veto. Ultra-clean materials were used in its construction, including electroformed copper. Care was taken to limit the cosmogenic exposure of the germanium following enrichment, fabricated detectors, and any electroformed copper components, which required above-ground fabrication processes. The resulting low backgrounds, coupled with low noise electronics, enable sensitive searches for other physics beyond the Standard Model [2, 3].

\section{Pulse shape based background rejection techniques}

In addition to shielding and ultra-clean material selection, analysis of detector pulses also provides a means to reject backgrounds. Two features of the p-type point contact detectors [1] are their range of drift times and localized weighting potential. Background $\gamma$-ray events often deposit energy at multiple points in the detector, while double-beta decays release their energy at a single site (within $\sim 1 \mathrm{~mm}$ of the event location). Through examination of the current pulse amplitude versus the total energy, multi-site events can be distinguished from single-site events and rejected.

Another technique concerns an observed energy-degraded ${ }^{210} \mathrm{Po}$ alpha background that studies indicate likely originates from ${ }^{210} \mathrm{~Pb}$ near the detector's point contact. This alpha must pass through the passivated surface of a detector, and the slow drift of charges along the passivated surface produces a distinctive waveform, allowing this background to be identified and rejected.

\section{Neutrinoless double-beta decay results}

The Majorana Demonstrator has been operating in a stable configuration since the spring of 2017. Changes between earlier configurations require the data to be split into multiple datasets (DS). In particular, there is higher background in DS0, taken prior to the installation of the inner copper shield; and in DS5a, when a single DAQ system was used and the pulse shape analysis was degraded by high electronic noise until the shielding and grounding path were completed. Therefore, DS0 and DS5a are excluded from the lowest background configuration, which better represents achieved background rates. Simulations and assays indicate that when prominent background $\gamma$ lines are excluded, the spectrum from $1950 \mathrm{keV}$ to $2350 \mathrm{keV}$ is flat in shape. Therefore, to estimate the background, a $\pm 5 \mathrm{keV}$ window is excluded around each of these lines $\left({ }^{208} \mathrm{Tl}\right.$ : $2104 \mathrm{keV}$; ${ }^{214} \mathrm{Bi}$ : $2118 \mathrm{keV}$, and $2204 \mathrm{keV}$ ), as well as $Q_{\beta \beta}=2039 \mathrm{keV}$ (the energy resolution achieved at $\mathrm{Q}_{\beta \beta}$ is $2.5 \mathrm{keV}$ FWHM). The remaining 360-keV window is used to estimate the background rate at $Q_{\beta \beta}$. To determine a limit on the $0 v \beta \beta$-decay half life, the $360-\mathrm{keV}$ window plus the $Q_{\beta \beta}$ region is analyzed with a nominal profile likelihood method, searching on top of a flat background for a peak at $Q_{\beta}$ modelled by an exponentially modified Gaussian distribution. Independent analyses are also peformed to cross-check the validity of the statistical result, using the Feldman-Cousins method, the CLs method (a modified profile likelihood method) and a Bayesian Markov-Chain Monte Carlo (with a flat prior assigned to the $0 v \beta \beta$-decay rate). 
In 2017 [4], open data in DS0-5b (9.95 $\mathrm{kg} \cdot \mathrm{yr}$ of enriched germanium exposure) was analyzed, finding a background rate of $17.8 \pm 3.6$ counts/(FWHM.t.yr), and $T_{1 / 2}^{0 v}>1.9 \times 10^{25} \mathrm{yr}$ (median sensitivity: $2.1 \times 10^{25} \mathrm{yr}$ ) at the $90 \%$ confidence level. There was $5.24 \mathrm{~kg} \cdot \mathrm{yr}$ of exposure in the lowest background configuration, which had a background rate of $4.0_{-2.5}^{+3.1}$ counts/(FWHM.t.yr) or $1.6_{-1.0}^{+1.2} \times 10^{-3}$ counts/(keV·kg.yr).

The MAJORAnA DEMONSTRATOR employed a statistical blindness scheme, whereby 31 hours of open data is followed by 93 hours of blind data with the full spectrum blinded. Unblinding was performed in three phases to enable data quality and consistency checks. First, the region outside the background evaluation window was opened in order to finalize the run and channel selection. Then, the background evaluation window was opened to measure the background. Finally, the $\mathrm{Q}_{\beta \beta}$ region was opened, and the half-life limit is set. Multi-detector events and the spectrum below $100 \mathrm{keV}$ remain blind for other studies.

For the 2018 analysis, all open and blind data taken before April 15, 2018 was analyzed. This added $16.1 \mathrm{~kg} \cdot \mathrm{yr}$ of exposure, for a total of $26 \mathrm{~kg} \cdot \mathrm{yr}$ of enriched germanium exposure. Analysis of this exposure found a background rate of $15.4 \pm 2.0$ counts/(FWHM.t.yr) and a half-life limit of $T_{1 / 2}^{0 v}>2.7 \times 10^{25} \mathrm{yr}$ (median sensitivity: $4.8 \times 10^{25} \mathrm{yr}$ ) at the $90 \%$ confidence level. The lowest background configuration had an enriched germanium exposure of $21.3 \mathrm{~kg} \cdot \mathrm{yr}$, and a background rate of $11.9 \pm 2.0$ counts/(FWHM $\cdot \mathbf{t} \cdot \mathrm{yr})$ or $(4.7 \pm 0.8) \times 10^{-3}$ counts/(keV $\left.\cdot \mathrm{kg} \cdot \mathrm{yr}\right)$.

\section{Conclusions}

The Majorana Demonstrator has completed construction, and has been taking data in its final configuration since the spring of 2017. With a combined (open+blind) exposure of $26 \mathrm{~kg} \cdot \mathrm{yr}$, the Majorana Demonstrator places a lower limit on the $0 v \beta \beta$-decay half-life of $2.7 \times 10^{25} \mathrm{yr}$ (median sensitivity: $4.8 \times 10^{25} \mathrm{yr}$ ) at the $90 \%$ confidence level. Ultimately, the MAJORANA DEMONSTRATOR expects to reach an exposure of 50-70 kg.yr, pushing its sensitivity to a half-life $\sim 10^{26} \mathrm{yr}$. With its goal of lower backgrounds, sensitivity beyond $10^{28}$ years will be possible with the next-generation tonne-scale experiment, LEGEND. Its first phase, the 200-kg LEGEND-200, will begin taking data in 2021, and will have sensitivity beyond $10^{27}$ years.

\section{References}

[1] Majorana collaboration, N. Abgrall et al., The Majorana Demonstrator Neutrinoless Double-Beta Decay Experiment, Adv. High Energy Phys. 2014 (2014) 365432 [1308. 1633].

[2] Majorana collaboration, N. Abgrall et al., New limits on Bosonic Dark Matter, Solar Axions, Pauli Exclusion Principle Violation, and Electron Decay from the Majorana Demonstrator, Phys. Rev. Lett. 118 (2017) 161801 [1612.00886].

[3] Majorana collaboration, S. I. Alvis et al., First Limit on the Direct Detection of Lightly Ionizing Particles for Electric Charge as Low as e/1000 with the Majorana Demonstrator, Phys. Rev. Lett. 120 (2018) 211804 [1801.10145].

[4] Majorana collaboration, C. E. Aalseth et al., Search for Neutrinoless Double- $\beta$ Decay in ${ }^{76}$ Ge with the Majorana Demonstrator, Phys. Rev. Lett. 120 (2018) 132502 [1710.11608]. 